\title{
Psychological Interpretation of Human Behavior According to External Circumstance Changes in Atypical Space
}

\author{
Young Lim Lee ${ }^{1}$, Yun Gil Lee ${ }^{2}$ \\ ${ }^{1}$ Assistant Professor, Department of Psychology \& Psychotherapy, Dankook University, Republic of \\ Korea,younglee13@dankook.ac.kr \\ ${ }^{2}$ Professor, Department of Architecture, Hoseo Univeristy, Republic of Korea, yglee@hoseo.edu
}

Corresponding author: Yun Gil Lee

\begin{abstract}
Atypical buildings can give users a variety of spatial experiences compared to existing typical buildings. Thus, the ability to design atypical structures has become a vital aspect for architects. Users interact not only with the physical context of atypical spaces and other users but also with changes in the surrounding context. External circumstance changes are diverse, such as weather, fire, riots, and gatherings. Thus, to realize user simulation in a more realistic atypical space, it is necessary to develop an intelligent agent that responds to changes in various external situations. This study is part of a research project on the development of intelligent agent technology that responds to changes in external circumstances. Specifically, users' behavioral changes according to external situation changes in architectural spaces were investigated, and the psychological mechanism was theoretically analyzed. In addition, according to the specificity of an atypical space, the difference in response behavior to changes in external circumstances was theoretically considered. Through on-site and media investigations, it was confirmed that users' actions responded to changes in external circumstances, and it was assumed that there was a psychological cause for this. Moreover, it was assumed that there would be differences in psychological changes and response behaviors due to changes in external circumstances because atypical spaces have different physical and social situations compared to typical architectural spaces. This study theoretically analyzed the psychological mechanisms of users' behavioral changes according to external situation changes in atypical architectural spaces. According to the specificity of an atypical space, the difference in response behavior to changes in external circumstances was also theoretically considered. And also, several strategies for the advancement of human behavior simulation can be derived. First, in the simulation of human behavior in an atypical space, the scope and method of the agent's perception should be adjusted based on the theory of direct perception so that the simulation can be highly realistic. Second, according to the positive and negative changes in the external situation, parameters that affect the agent's reaction behavior, such as pathfinding, should be developed and applied to the simulation. Third, it is necessary to develop an environment that can perform human behavior simulation by making changes in external circumstances within the design and developing agents that respond in various ways. The results of this study are meaningful, as they can be used as a basis for future human behavior simulation and to advance agent intelligence.
\end{abstract}

Keywords: Atypical Architecture, Human Behavior Simulation, External Context Change, Psychological Interpretation

Received: September 4, 2021; $1^{\text {st }}$ Review Result: October 20, 2021; $2^{\text {nd }}$ Review Result: December 6, 2021 Accepted: January 29, 2022 


\section{Introduction}

As various desires in life increase, atypical space design is also increasing. Atypical buildings can give users a variety of spatial experiences compared to existing typical buildings. Thus, the ability to design atypical structures has become a vital aspect for architects. Additionally, architectural design tools also need to support the design process of atypical buildings, and these tools are being developed and distributed. Nowadays, theories and technologies for designing atypical buildings are taught in most architectural education sites[1].

Unlike traditional architectural design, atypical architectural design creates a variety of spaces that have not been seen before. Atypical buildings are difficult to construct compared to existing typical buildings due to the specificity of their shape. Consequently, technical problems such as structure, equipment, and envelope have been the main issues raised. To date, many studies have been conducted with the goal of seeking technical solutions for the design and construction of atypical buildings[2].

In this study, the researchers focused on the more fundamental problems of atypical buildings related to the value of these buildings. Specifically, this study is concerned about the convenience and safety of the building's users. Due to the morphological peculiarities of atypical buildings, architects may sometimes overlook the users of the building. To solve this problem, research on human behavior simulation based on atypical design tools is in progress. Prior research found that these tools help architects to improve user safety and convenience in the design process and derive more diverse creative results[1]. [Fig. 1] shows the results of user simulation in an atypical space in a previous study[3].



[Fig. 1] Examples of Human Behavior Simulation in the Atypical Design Process 
The behavior of users in a real atypical space is shown in [Fig 2]. Here, it should be noted that users not only interact with the physical context of an atypical space and other users but also with changes in the surrounding context. These external circumstance changes are diverse, such as weather, fire, riots, and gatherings. Therefore, to realize user simulation in a more realistic atypical space, it is necessary to develop an intelligent agent that responds to changes in various external situations.

This study is part of a research project on the development of intelligent agent technology that responds to changes in external circumstances. The purpose of this study is to examine the psychological mechanism of behavior change in response to external situation change and apply it to agent intelligence. The researchers conducted the research with the following research questions. (1) Through on-site and media investigations, it is confirmed that users' actions responded to changes in external circumstances, and it is assumed that there was a psychological cause for this. (2) It is assumed that there would be differences in psychological changes and response behaviors due to changes in external circumstances because atypical spaces have different physical and social situations compared to typical architectural spaces.

This study investigated users' behavioral changes according to external condition changes in the architectural space and theoretically analyzed the psychological mechanisms. According to the specificity of an atypical space, the differences in response behavior to external circumstance changes were also theoretically considered. The results are meaningful, as they can form the basis of future human behavior simulation and advance agent intelligence.



[Fig. 2] Human Behaviors Reacting to External Situation Changes in an Atypical Architectural Space 


\section{Human Behaviors Reacting to External Context Changes in Atypical Space}

Physical space defines human behavior. Additionally, behavioral changes occur in interactions with others. Most existing human behavior simulation technologies target interactions between human behavior and the physical environment. Some human behavior simulation techniques consider social interactions as well as physical environments. [Fig. 3] shows users' reaction behavior to changes in external circumstances in a space derived through field and media surveys. It highlights further considerations that are needed to refine human behavior simulation. Looking at [Fig. 3], you can see how users respond to various external situation changes such as fire, rainfall, snowfall, earthquake, terrorism, and small groups. In the existing human behavior simulation technology, technologies for simulating the user's escape are developed and used by specializing in fire situations. However, the possibility that the user's behavior may be changed through a change in the situation is not seriously considered. Changes in external circumstances are highly likely to cause psychological changes in users, which can lead to changes in user behavior[4]. In other words, understanding and applying such external situation changes and users' psychology will further advance human behavior simulation technology.

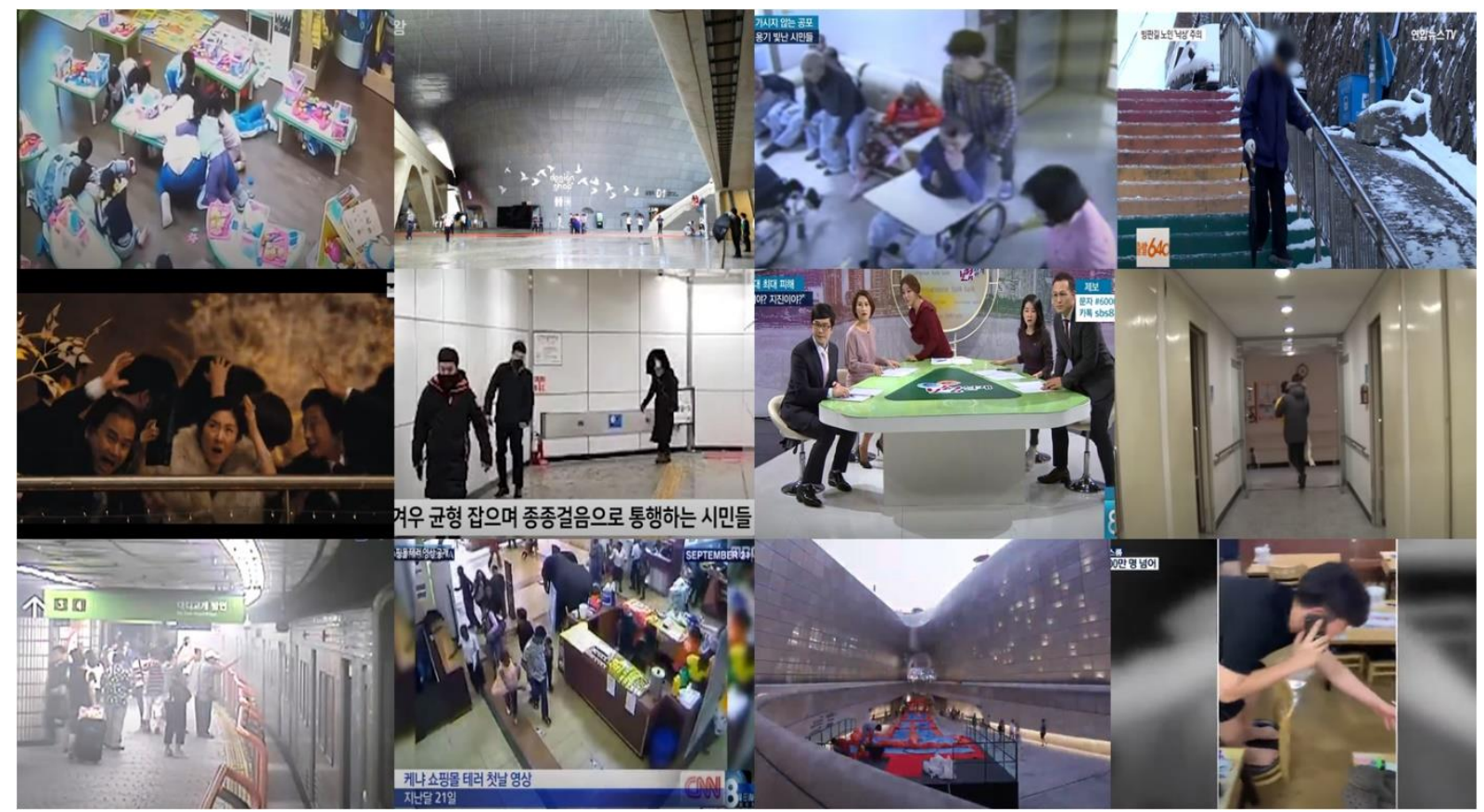

[Fig. 3] Users' Reaction Behavior to Changes in External Circumstances in the Built Environment

\section{Psychological Analysis of Human Behaviors According to External Circumstance Changes}

\subsection{Methods}

In this study, human behavior in response to changes in the external environment in an atypical architectural space was analyzed based on psychological theories. Because the user's behavior in the architectural space is achieved through psychological perception and cognition, this study was focused on $3 \mathrm{D}$ perception and the psychological response to changes in the external environment. The research was conducted in a way that related contents were derived through theoretical analysis and strategies 
for simulation were established.

First, the researchers explored perception, which is closely related to behavior. In particular, through the psychological theories regardoing three-dimensional (3D) perception, we investigated how the user perceives the space in an atypical space. The reason why the 3D perception was considered first is that people take corresponding actions after they perceive an object. Therefore, depending on the perception method or procedure for the object, the response behavior would be different. In fact, vision, plays a critical role in our movement or action by giving us the information about the world. Second, the psychological interpretation of users' perception of atypical spaces was explained based on indirect and direct perception theory.

In this section, users' reactions to external situation changes in atypical spaces were explained based on psychological theories. First, the researchers examined the concept of 'affordance' in order to examine the difference in the method of perceiving an object. Second, because changes in external circumstances are recognized through visual attention and users act in response to them, theories related to 'visual attention' were considered. Last, unexpected changes in external circumstances and psychological responses and behaviors were discussed.

\subsection{Psychological Theories of Three-Dimensional Perception}

\subsubsection{Indirect Versus Direct Perception}

The user's actions in the physical space are based on the information processing of perception. In other words, environment must be perceived to take actions. Therefore, to psychologically understand the user's behavior in the physical space, it is necessary to consider the relationship between perception and action. Since humans perceive the 3D environment based on 2D retinal information, how users perceive 3D space is not an easy question to answer. Thus, this has become an important topic of psychological research. The most representative psychological theories of 3D perception are indirect and direct perception[5].

Traditionally, the psychological interpretation of human 3D perception has been centered on indirect perception or causal theory. Causal theory defines visual perception as sensation mediated perception. This theory starts with assumptions about the machine to determine input. From this theory, our eye functions like a camera and it takes static pictures called retinal image. The picture is registered in the back of the eye. This "registration" is nothing more than an arrangement of points reflecting different wavelengths and intensities. That picture is transmitted to the brain by specific nerve energies. Physically, there are no colors, shapes, odors, counds, and so on, but these subjective impressions of the scene are the result of inserted meaning in the process of perception through sensation. The particular shape of the retinal image is greatly variable as we move from one position to another. Despite this lack of correspondence between the retinal image and te actual 3D space, our perception remains remarkably stable and accurate. To recognize and identify the variable actual space to the constant retinal image, past experiences carried over in our memory must be used. To do that, the brain must integrate several static pictures taken by a stationary eye after eye moves to one by another moment. In short, the perceived object or space seems to be the result of a process of construction over time[6]. This can be explained by the fact that humans can take actions in familiar spaces even with their eyes closed. In addition, indirect perception can be understood by the example of being able to find directions more easily in subway spaces that you usually visit. Conversely, direction perception is not based on the having of sensations, rather on the pickup of information. According to indirect perception theory, perception is to copy the physical world and our memory has a role of perception when the object is out of sight. According to direct perception theory, on the other hand, optic array information need not resemble the physical world and the information of persistence lies not in the persistence of stimulation, but in specific patterns of change. When we have a motion or see object 
motion, the surfaces of objects are occluded or hidden by other surfaces. These surfaces in world is not going out of existence, but rather going out of sight because of the deletion of optical texture at an edge. The optical texture trnasformations are reversible because deleted texture is going to come into the sight again when the optical texture at the ende is accreted. So optical texture transformation is not similar to surface (world) texture transformation. In short, the perception of 3D is made directly through incoming visual information and is frequently corrected through actions to achieve more accurate $3 \mathrm{D}$ perception. In direct perception, the perceiver is active in exploring the contents of the environment (seeing, feeling, smelling, tasting, hearing). Thus, the perceiver is an active and purposeful acquirer of information, not a passive information receiver. If the information is poor, the normal and active perceiver is preoccupied with activities to obtain more information[5]. From the view of direct perception, environment is not physical, but ecological. Ecological psychology emphasizes the relationship between people and the environment; its basic premise is that movement is caused according to the characteristics of the environment. Therefore, ecological theory asserts that the environmental context is important and different environmental contexts create different movements for different purposes[4].

\subsubsection{Three-Dimensional Perception in the Simulation Environment}

Indirect perception theory suggests that $3 \mathrm{D}$ space is perceived by constructing a lot of individually sampled information with the mediated cognitive process using memory. Direct perception theory, on the other hand, suggests that 3D space is perceived through the interaction of visual information directly picked up and action. As the mechanism of direct perception is not easily simplified, it is difficult to access. In general, it is often thought that 3D space is perceived by the method of indirect perception. As an example of human behavior simulation in the field of architecture, research is often conducted based on the concept of indirect perception. A typical application field of human behavior simulation is escape simulation. Traditionally, the A* algorithm or Dijkstra's algorithm is often used as the pathfinding algorithm used in the escape simulation. In the case of this algorithm, the pathfinding operation is performed in a state where the structure of the space is known to some extent. In other words, the psychological background for the basis of the perception and behavior of space in an escape simulation can be considered in line with indirect perception. However, there are many games in which the characters are set to understand the space in a limited manner and perform pathfinding as they move. They directly acquire information about space through dynamic movement (action) in space and increase their understanding of space through feedback. This can increase the realization and dynamism of the game.

\subsubsection{Spatial Perception and Human Behavior in the Atypical Space}

Spatial perception in an atypical space can be considered based on the theories of indirect and direct perception. Atypical spaces have many differences in form from typical architectural spaces. Users are not familiar with this type of 3D space. This is because buildings with atypical spaces are rare, so people do not usually experience them frequently, and the irregular space is not a generalized form because the behavior itself is free and diverse.

If the user's perception of space in an atypical space is interpreted based on the indirect perception theory, it can be said that the user has a very difficult state to perceive space correctly. This is because sufficient information about the atypical space has not been acquired. This may be similar to the user's situation in an unfamiliar space. However, the user may be more familiar with the form of a modern, typical space. Therefore, based on the theory of indirect perception, users will have more difficulty in responding to space or finding directions in an atypical space. 
Based on the direct perception theory, it can be said that the interpretation of the user's perception of space in an atypical space is not much different from the perception of space in a typical space. Of course, the response behavior might be different because the user's situations in that space are different. Nontheless, the method or degree of perceiving a 3D space may not be different because no top-down cognitive process integrating with visual information is needed. Naturally continuing actions, even in unfamiliar spaces such as atypical spaces, can keep giving a feedback on perception and calibrating visually-guided actions.

\subsection{Human Behavior According to External Situation Changes in the Atypical Space}

\subsubsection{Affordances}

To psychologically interpret response behavior to changes in the external environment, it is necessary to first understand the concept of affordance. Affordance is a behavior induced by an object, place, or event. The motivating properties of the environment, good or bad, are what the environment provides to animals[7]. For example, chairs, benches, and stools induce sitting; a subject with a handle leads to grab it; cliffs lead avoiding; the bottle induces drinking and, alternatively, throwing. In its simplest form to evoke action refers to what meaning the environment gives to the perceiver. What causes a water bottle to grab is that when we perceive a water bottle, humans know how to grab it with their hands. According to Gibson, affordances, such as "sti-on-able" or "climb-on-able", are specified in the stimulus information for users. When perception is interpreted in this way, we can say that we do not perceive chairs, pencils, and donuts per se. We perceive where we will sit, what we will have, and what we will eat. To say that an affordance is perceived means that the information for specifying the behavior inducement is available in these stimuli and can be detected by an appropriately coordinated perceptual system. Thus, detecting causality is simply detecting meaning.

Even if all the information is the same, different perceivers pay attention to different invariants. There is information that specifies, for example, whether a bottle is for drinking or throwing. Diversity of perception is expected because different perceivers tend to select information for various reasons. It can also be expected that the same perceiver can detect different information under different conditions. The source of diverse experiences lies in the diversity of stimuli. Gibson called this process of selecting input "information attention". Exploratory activity is the type of attention process. In exploratory activity, perceivers, in general, actively search their environment for a specific purpose. For instance, we can lift a stone to see if it is large enough to throw. Information that can be detected by searching for appropriate or detailed parts of the environment can be controlled.

Such detection is a direct and goal-oriented proposal in terms of affordance. Affordance relates the environment to the actor-perceiver, for example, in terms of the uses the animal has for it, whether to throw or eat it. To benefit the animal, information specifying the behavioral tendency must be detected. As an exploratory activity, attention reveals affordances in animals[4]. When a stone is lifted, people can perceive whether to throw it or not. For example, if there is a flood and I climb on top of a structure, I will find a structure that can hold up, or I will press the structure with an arm and search for a structure that is safe enough to support me. The structure will evoke the action of climbing to escape from the flood, not its original function.

\subsubsection{Visual Attention}

Our attention to a certain position is caused by an involuntary process in which a striking stimulus grabs attention, or by a voluntary process according to our goals and intentions. Visual salience refers to physical properties (such as color, contrast, movement, orientation, etc.) that make a specific object or location stand out. When an involuntary transfer of attention occurs due to stimulus salience, this 
transfer is called attentional capture[8]. For example, a bright flash or loud sound can distract our attention from what we were doing. This attentional capture can be important if it serves to warn of danger, such as an explosion, dangerous animal, or fast-moving object coming toward us. Therefore, if there is a big fire or a gunshot is heard, it is necessary to catch the attention and avoid the danger. However, gaze initially occurs in areas of high salience, but after a few gaze points, it begins to be influenced by top-down or cognitive processes that depend on something like the observers' goals or expectations. These goals and expectations are influenced by the observer's past experiences in environmental observation. Top-down processing involves the scene schema as the observer's knowledge of what will be in the formal scene. Vo and Henderson showed the observer a scene of the kitchen, one with a pot and the other with a printer. The observer spent more time looking at the printer than the pot because the printer is less likely to be in the kitchen[9].

The fact that people spend longer looking at things that seem inappropriate in a scene means that attention is influenced by their knowledge of what is usually found in the scene[10]. In the event of a small fire at a subway station, people pay attention because something inappropriate happened in the scene. Paying attention tells us what is happening in a situation and also allows us to react more quickly to anything happening in that situation. Therefore, in the event of a small fire, attention has been paid to it, so when it leads to a large fire, you can quickly react and escape.

\subsubsection{Psychological Response and Behavior toward Unexpected Situations}

An external situation change can be divided into a positive or negative situation. That is, there are external circumstance changes that do not adversely affect oneself, such as pleasant sounds, songs, and children playing, and there are external situation changes that are highly likely to have a bad effect on oneself and others, such as fire, terrorism, and bad weather. This situation can be interpreted by the theories of indirect perception, direct perception, affordance, and visual attention discussed above. In addition, it can be an important foundational theory for the development of human behavior simulation technology.

It can be said that the emergence of a positive situation raises visual attention and suggests the possibility of triggering other behaviors. The user may perform a natural reaction behavior toward the place where the positive situation appeared, that is, simply turn their head to look or approach. Otherwise, they may ignore the contingency and continue the previous behavior. A negative situational appearance can be seen psychologically as the same mechanism as a positive situational appearance in generating visual attention and suggesting a falsehood that induces other behaviors. However, the appearance of a negative situation is different because it requires quick judgment and an active response from the user. There is also a difference in proceeding avoiding rather than approaching the situation that emerged. For example, in the event of a fire, you need to get out of the building as quickly as possible. In an escape situation, the action can be considered to be made through indirect perception. In other words, the user finds an evacuation route and acts through the information obtained in advance. Many of the existing simulation technologies have been developed based on such psychological situations. However, what we are interested in here is the perception of space in an unfamiliar space, such as an unfamiliar space or a field of view covered by smoke. In such a case, it is not possible to use the spatial perception method suggested by the indirect perception theory. In other words, as claimed by the theory of direct perception, a reaction is made while perceiving the visible spatial situation, and the perception and action interact by receiving feedback accordingly. To acquire the spatial information necessary for escape, the person will constantly act or move their gaze. Ultimately, through this process, an appropriate escape route may or may not be found.

There may be factors influencing the response behavior toward the appearance of negative situations in such an atypical space. One of them is the misunderstanding of space. That is, there are 
cases in which the form of the space shown is not an appropriate escape route to confuse the user. Another thing is the behavior of others. This refers to imitating the escape behavior and direction of others in a state where the perception of escape has not been sufficiently achieved[11]. This problem can be reduced to some extent if the emergency escape routes are clearly marked. Furthermore, the altruistic behavior of helping others may occur. In an atypical space, there are more situations in which movement is unfavorable to the walking weak, such as the slope of a curved passageway, so the possibility of altruistic behavior is high. It is difficult to generalize because there are differences in individual values, but the norms for social responsibility are the cause of determining behavior[12].

\section{Discussion and Conclusions}

Unlike human behavior simulation in a typical architectural space, user behavior simulation in an atypical space requires the specificity of the spatial form and its application to the situation. In this study, changes in external situations and corresponding behaviors were considered psychologically to serve as a theoretical basis for simulation technology. Through these psychological considerations, several implications for sophisticated human behavior simulation could be drawn. First, the perception of 3D space is generally explained by the theory of indirect perception in psychology, but the form of perception in an atypical space is often related to direct perception. Second, it can be said that the perception of changes in external circumstances is the same in positive and negative situations, but the response behavior may appear different. Third, in the perception and response to negative external situation changes, psychologically different aspects can be seen in an atypical space, unlike in a typical space.

From these observations, several strategies for the advancement of human behavior simulation can be derived as follows. First, in the simulation of human behavior in an atypical space, the scope and method of the agent's perception should be adjusted based on the theory of direct perception so that the simulation can be highly realistic. Second, according to the positive and negative changes in the external situation, parameters that affect the agent's reaction behavior, such as pathfinding, should be developed and applied to the simulation. Third, it is necessary to develop an environment that can perform human behavior simulation by making changes in external circumstances within the design and developing agents that respond in various ways. Through this, it is possible to review the performance of the user side of the designed atypical space in real time.

This study theoretically analyzed the psychological mechanisms of users' behavioral changes according to external situation changes in atypical architectural spaces. However, there is a limitation in not being able to prove whether these psychological mechanisms are equally applied in real situations. This needs to be proven through future field investigations and analysis. According to the specificity of an atypical space, the difference in response behavior to changes in external circumstances was also theoretically considered. It is significant that the theoretical basis for developing advanced human behavior simulation technology in an atypical space was secured through the considered psychological theory. However, it is necessary to prove the effectiveness of the theory through technological development and experiments. In addition, it is necessary to investigate what effect the developed system has on the atypical design process.

\section{Acknowledgments}

This work was supported by the National Research Foundation of Korea (NRF) grant funded by the Korea government (MSIT) (NRF-2018R1A2B6005827). 


\section{References}

[1] Y. G. Lee, The Effect of Human Behavior Representation on Creative Motivation in Atypical Architectural Design in Architectural Design Studio, Asia-pacific Journal of Multimedia Services Convergent with Art, Humanities, and Sociology, (2019), Vol.9, No.6, pp.203-210, DOI: 10.35873/ajmahs.2019.9.6.019

[2] S. J. Park, A Study on the Convergence Relativity of the Combining Curved Forms of Tall Buildings, The Journal of the Korea Contents Assoication, (2020), Vol.20, No.3, pp.190-199, DOI:10.5392/JKCA.2020.20.03.190

[3] J. M. Park, H. S. Lee, S. J. Kim, Y. G. Lee, Developing a Deployment Technology for Virtual Users with an Autonomous Psychological Behavioral Simulation in Atypical Architectural Space, HCI International 2020 - Posters, Springer, pp.217-222, (2020), July 19-24, Copenhagen, Denmark, DOI: https://doi.org/10.1007/978-3-030-507299_31

[4] H. Heft, Ecological psychology in context: James Gibson, Roger Barker, and the legacy of William James's radical empiricism, Psychology Press, pp.109-142, (2005)

[5] C. F. Michaels, C. Carello, Direct perception, Prentice-Hall, pp.15-35, (1981)

[6] I. Rock, The perceptual world: readings from Scientific American magazine, W H Freeman, pp.9-18, (1989)

[7] J. J. Gibson, The Ecological Approach to Visual Perception, 1st Edition, Psychology Press, pp.127-143, (1986)

[8] B. A. Anderson, P. A. Laurent, S. Yantis, Learned value magnifies salience-based attentional capture, PloS one, (2011), Vol.6, No.11, e27926, DOI: 10.1371/journal.pone.0027926

[9] M. L. H. Vo, J. M. Henderson, Effects of semantic and syntactic object-scene inconsistencies on the allocation of attention during naturalistic scene viewing, Journal of Vision, (2009), Vol.9, No.8, p.418, DOI: 10.1167/9.8.418

[10] E. B. Godstein, Sensation and Perception, International Edition, 9th Edition, Cengage Learning, pp.145-157, (2013)

[11] D. G. Myers, Social psychology, 11th Edition, McGraw-Hill Education, pp.226-260, (2013)

[12] D. G. Myers, Social psychology, 11th Edition, McGraw-Hill Education, pp.521-531, (2013) 